\title{
Chronisch intestinale Erkrankungen
}

D ie aktuelle Ausgabe des klinikarzt widmet sich verschiedenen Aspekten der Gastroenterologie und Hepatologie. In ihren Beiträgen stellen die Autoren neue Erkenntnisse und interessante Zusammenhänge zusammen und bieten Leitfäden für Diagnostik und Therapie.

Die Obstipation zum Beispiel ist ein wissenschaftlich wenig beachtetes Krankheitsbild, in der Bevölkerung ist sie jedoch weit verbreitet und führt die Patienten häufig zum Arzt. Prof. J.F. Erkenbrecht, Düsseldorf, vermittelt in seinem Artikel einen systematischen Überblick über die verschiedenen Formen der Obstipation. Hervorzuheben ist der Abschnitt über die Diagnostik, die - obwohl sehr einfach - im Klinikalltag oft nicht voll ausgeschöpft wird, woraus Fehldiagnosen resultieren können. Die Autoren geben wertvolle Tipps zur Behandlung der hartnäckigen Obstipation.

Ein weiterer Beitrag befasst sich mit Cholangiopathien: PD Chr. Elsing, Dorsten, hat darin zellbiologische Aspekte, die zur Pathogenese verschiedener Gallenwegserkrankungen beitragen, zusammengefasst. Von diesen molekularen Mechanismen werden therapeutische Strategien abgeleitet. Wer weiß schon, dass die Funktionsstörung der Zilien auf Cholangiozyten die Entwicklung von Leberzysten bahnt, oder dass durch Xenobiotika-Modifikation des E2-Komplexes der mitochondrialen Pyruvat-Dehydrogenase der Auslösemechanismus für die primäre biliäre Zirrhose (PBC) als Autoimmunerkrankung getriggert wird? In dem Artikel wird abgeleitet, wie die Gallengangsschädigung zustande kommt, wie sich die Graft-versus-Host-Erkrankung (GvHD) an Gallenwegen manifestiert, und warum Ursodeoxycholsäure therapeutisch wirksam ist. Dass die HCV-Infektion Gallenwege zerstört und sich die Viren so in der Leber ausbreiten oder warum bestimmte Medikamente zur Cholangitis führen, wird hier ebenfalls beschrieben. Der Beitrag erklärt die Entstehung der ischämischen Cholangiopathie als eigenständiges Krankheitsbild. Darüber hinaus wird erläutert, wie Gallengangskarzinome entstehen könnten und welche therapeutischen Strategien eingeleitet werden können.

In seiner Arbeit zur chirurgischen Behandlungsstrategie bei chronisch entzündlichen Darmerkrankungen bezieht PD B. Eibl-Eibesfeldt, Nürnberg, systematisch und klar zu den aktuell empfohlenen Operationsverfahren Stellung. Neben den Vorteilen beleuchtet er kritisch die Komplikationsraten und beschreibt - nach dem neuesten Stand der Technik - einen praktikablen Algorithmus zur operativen Behandlung chronisch entzündlicher Darmerkrankungen. Inhalte seiner Arbeit sind die Indikation zur Operation, die operative Technik, die Operationsergebnisse und letztendlich auch die Lebensqualität des operierten Patienten. Dieser ausgewogene Artikel eines erfahrenen Chirurgen kann Empfehlung für jeden klinisch tätigen Arzt sein.
Um die nichtalkoholisch bedingte Fettleberhepatitis (NASH) wird in den letzten Jahren viel Aufmerksamkeit gebündelt, obwohl das Prinzip der Fettüberladung in der Leber schon lange bekannt ist. Bis heute sind die pathophysiologischen und pathogenetischen Prinzipien der Fettleber jedoch nicht vollständig aufgeklärt. Einer der wichtigsten Faktoren für die Entstehung der NASH häufig als Erkrankung der Wohlstandsgesellschaft interpretiert - scheint die Insulinresistenz zu sein. In den industrialisierten Ländern ist eine ständig steigende Inzidenz der Fettleber festzustellen. Wichtig ist die Unterscheidung zwischen alkoholischer und nichtalkoholischer Genese, um die ungerechte Einstufung eines Patienten als Alkoholiker zu vermeiden bzw. die zu erwartende Prognose besser einschätzen zu können. Dr. M. Karner, Heidelberg, weist in seiner Arbeit auch auf das Dilemma der fehlenden bzw. unzureichenden Therapiestudien hin, die den Standards der evidenzbasierten Medizin noch nicht entsprechen. Bei den meisten Studien sind die Patientenzahlen sehr gering, die Beobachtungsdauer zu kurz oder das Design entspricht nicht den Kriterien einer optimalen Studienplanung. Allerdings verweist er auf eine neue, gut konzipierte Studie von Lindor und Mitarbeitern, die keinen positiven Effekt von Ursodeoxycholsäure auf den Verlauf der Erkrankung zeigte. Damit wird eine landläufig geübte Therapie sehr infrage gestellt.

Das hier vorliegende Heft ist inhaltlich zum Teil provokativ, neu, systematisch, praxisorientiert und somit eine wertvolle Informationsquelle für klinisch tätige Ärzte.

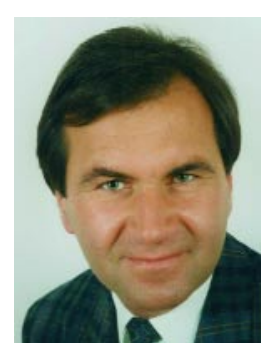

Prof. Dr. W. Stremmel, Heidelberg

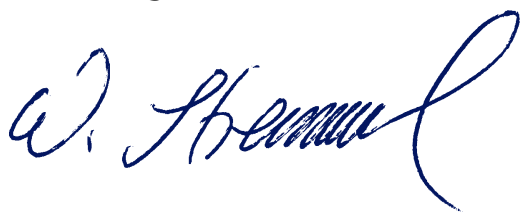

